\title{
The Synthesis and Properties of High Surface Area $\mathrm{Fe}_{2} \mathrm{O}_{3}$ Materials
}

\author{
Z. SurowieC ${ }^{a, *}$, W. GAC ${ }^{b}$ AND M. Wiertel ${ }^{a}$ \\ ${ }^{a}$ Institute of Physics, M. Curie-Skłodowska University, pl. M. Curie-Skłodowskiej 1, 20-031 Lublin, Poland \\ ${ }^{b}$ Faculty of Chemistry, M. Curie-Sklodowska University, pl. M. Curie-Skłodowskiej 1, 20-031 Lublin, Poland \\ Two types of the silica materials were used for the preparation of iron oxides. The MCM- 48 type silica support \\ and wide-pore silica gel were impregnated with iron nitrates. The samples were investigated by the nitrogen \\ adsorption/desorption method, X-ray diffraction, scanning electron microscopy, and ${ }^{57}$ Fe Mössbauer spectroscopy. \\ Redox properties were studied by the temperature programmed reduction method. Strongly dispersed hematite \\ was evidenced on the supports. An application of the MCM- 48 silica mesoporous materials led to the development \\ of the oxide phases within the ordered porous structure. The detailed temperature programmed reduction studies \\ evidenced slight differences of the redox properties of the species located within the silica templates and after \\ their removal. Magnetic properties of iron oxides were related to the porous structure of the silica supports. \\ The Mössbauer study of high surface area materials $\mathrm{Fe} / \mathrm{SiO}_{2}$ and $\mathrm{Fe} / \mathrm{MCM}-48$ type silicate templates revealed \\ existence of ferro- and superparamagnetic phases. The relative contribution of the superparamagnetic doublet \\ component in Mössbauer spectra is $15 \%$ and $80 \%$ for $\mathrm{Fe} / \mathrm{SiO}_{2} / \mathrm{c}$ and $\mathrm{Fe} / \mathrm{MCM}-48 / \mathrm{c}$ samples, respectively.
}

PACS: 76.80.+y, 78.67.Bf, 78.67.Rb, 75.20.--g

\section{Introduction}

Nanostructured iron oxides have found widespread interests in recent years [1-4]. Iron oxides and iron-based materials reveal often unique physical, optical, magnetic and catalytic properties. These relatively cheap and nontoxic materials have many high-technology potential applications. They can be used for example for enhanced storage media $[5,6]$, in biosensing, as contrast agents in magnetic resonance imaging [7] or drug and gene delivery [8], as ferrofluids [9] or catalysts [10]. The coercivity, saturation magnetization values of nanostructured iron oxides are strongly related to the size and magnetic anisotropy of the particles [11, 12].

The properties of iron oxide species can be tailored by the application of the nanocasting technique. In the first stage of this technique, metal ions are introduced to the solid matrices (such as carbon materials or silica support), then precursors are transformed to the larger species, and finally the matrices are removed by combustion or dissolution. Surface properties and porosity of silica support, initial concentration of metal ions, treatment temperature or the way of separation and stabilisation of the oxide nanoparticles may strongly influence their final properties.

The aim of the presented studies was preparation and investigation of the properties of iron oxide materials by the application of the nanocasting technique.

* corresponding author; e-mail: zbigniew.surowiec@umcs.lublin.pl

\section{Experimental}

The nanocrystal iron oxide was prepared in the high surface area silica materials. As-prepared MCM-48 type silica support and wide-pore silica gel were impregnated with the ethanol solution of iron nitrate. The samples $\mathrm{Fe} / \mathrm{MCM} 48 / \mathrm{c}$ and $\mathrm{Fe} / \mathrm{SiO} 2 / \mathrm{c}$ were dried and calcined. Next in both cases silica templates were dissolved in $\mathrm{NaOH}$ solution. The oxide was separated by centrifugation. Samples after drying were investigated by the nitrogen adsorption/desorption method and scanning electron microscopy. The nature of iron-oxygen interactions was studied by the temperature programmed reduction (TPR) method. Phase analysis was carried out by means of X-ray powder diffraction (XRD) with nickel-filtered $\mathrm{Cu}$ $\mathrm{K}_{\alpha}$ radiation. The Mössbauer spectra were recorded using a constant acceleration spectrometer with a ${ }^{57} \mathrm{Co}(\mathrm{Rh})$ source at room temperature. The Mössbauer spectra for ferrimagnetic phases were analysed by solving the complete Hamiltonian under the assumption that the energy of the quadrupole interaction is small compared to the hyperfine magnetic splitting. The fast-relaxation limit within the thin absorber approximation was accepted.

\section{Results and discussion}

The morphology of the samples is illustrated in the Fig. 1. The outer shape of the grains before and after silica removal is similar. However the Fe/MCM48 based oxides reveal more amorphous nature.

The total surface area of iron modified silica gel measurement by means nitrogen adsorption/desorption 


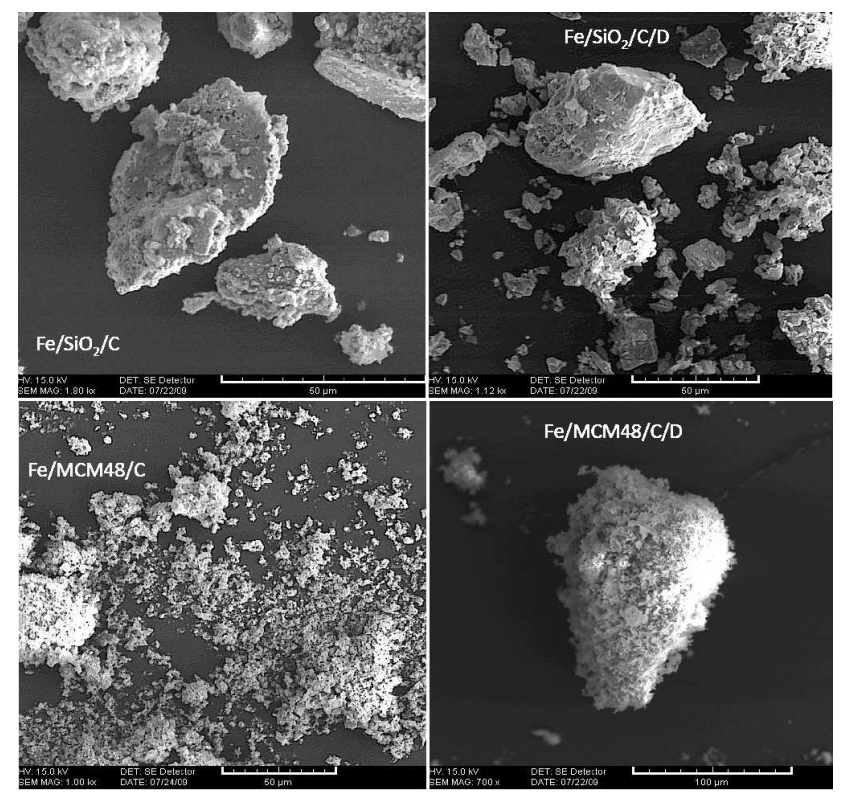

Fig. 1. Scanning electron microscope (SEM) images of the samples.

method is around $330 \mathrm{~m}^{2} / \mathrm{g}$. The sample shows wide pore size distribution. Mean pore diameter is in the range of $3.5 \mathrm{~nm}$. Nitrogen adsorption/desorption studies evidenced typical mesoporous structure of the MCM48 silica support and supported oxide materials (Fig. 2a). MCM48 type silica materials are characterised with narrow, interconnected uniform channels with the size in the range of $3 \mathrm{~nm}$ [13]. Such pre structure facilitates uniform deposition of iron species within the grains of the support. Samples after silica removal show lower surface area. The shape of the isotherm for $\mathrm{Fe} / \mathrm{SiO} 2 / \mathrm{c} / \mathrm{d}$ sample shown in Fig. 2b may indicate the presence of relatively large interparticle slit-like pores. Whereas Fe/MCM48/c/d sample may contain ink-bottle-like and wide interparticle pores. All parameters obtained by means nitrogen adsorption/desorption method for the both samples are collected in Table.

TABLE

BET surface area, total pore volume and pore diameter. $\mathrm{c}$ - samples after impregnation and calcination, $\mathrm{d}$ samples after removal of silica.

\begin{tabular}{l|c|c|c}
\hline \hline Sample & $\mathrm{S}_{B E T}\left[\mathrm{~m}^{2} / \mathrm{g}\right]$ & $\mathrm{V}_{t}\left[\mathrm{~cm}^{3} / \mathrm{g}\right]$ & $\mathrm{D}_{B J H}[\mathrm{~nm}]$ \\
\hline $\mathrm{Fe} / \mathrm{SiO} 2 / \mathrm{c}$ & 331.3 & 0.23 & 3.5 \\
$\mathrm{Fe} / \mathrm{SiO} 2 / \mathrm{c} / \mathrm{d}$ & 36.8 & 0.11 & 10.9 \\
$\mathrm{Fe} / \mathrm{MCM} 48 / \mathrm{c}$ & 742.2 & 0.50 & 2.5 \\
$\mathrm{Fe} / \mathrm{MCM} 48 / \mathrm{c} / \mathrm{d}$ & 26.8 & 0.04 & 4.9 \\
$\mathrm{MCM} 48$ & 1283.1 & 0.93 & 2.4
\end{tabular}

Temperature programmed reduction studies presented in Fig. 3 are often used to determine the oxidation state and slight differences of the metal-oxygen interactions,

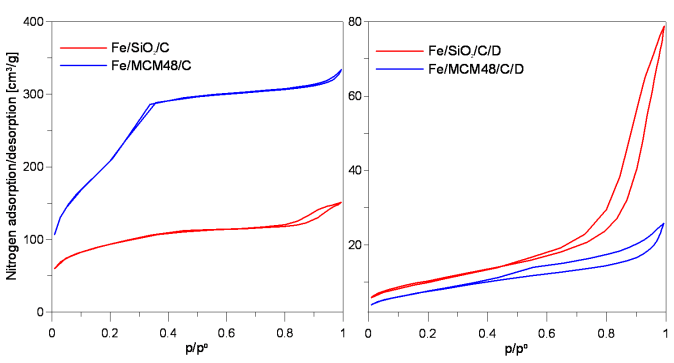

Fig. 2. Nitrogen adsorption/desorption isotherms (a) samples after impregnation and calcination; (b) samples after removal of silica.

which may results from different location of the species or interaction with the support. The first peak in the iron support oxides can be ascribed to the reduction of $\mathrm{F}^{3+}$ to $\mathrm{Fe}^{2+}[14]$. The second peak is often ascribed to the successive reduction of the oxides, which show different interaction with the iron support. The reduction of the samples after silica removal is more difficult. There are observed few peaks, which may indicate on the presence of different metal-oxygen interactions in the samples.

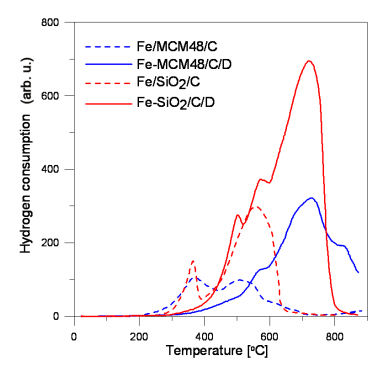

Fig. 3. Temperature programmed reduction profile.

The XRD patterns of the investigated samples are presented in Fig. 4. Analysis of the patterns evidences formation of the $\mathrm{Fe}_{2} \mathrm{O}_{3}$ in the both examined templates. The lattice parameters $a$ and $c$ of hematite nanocrystallites formed in $\mathrm{SiO}_{2}$ template are equal to 5.033(2) $\AA$ and $13.735(2) \AA$, respectively. These parameters are slightly smaller than those in case the bulk material $(\mathrm{a}=5.038 \AA$ and $\mathrm{c}=13.772 \AA$ ). The low intensity of the diffraction lines related to $\mathrm{Fe} / \mathrm{MCM} 48 / \mathrm{c}$ sample may indicate that hematite crystalline is strongly dispersed in the template.

In the presented study Mössbauer spectroscopy (MS) was used to confirm the formation of nanostructured iron oxides in the MCM-48 type silica support and wide-pore silica gel and stability of them after templates removal. The Mössbauer spectra are presented in Fig. 5. On the basis of the obtained hyperfine parameters values the presence of hematite in two magnetic states: ferromagnetic and superparamagnetic can be stated.

It is know that in nanocrystalline material the superparamagnetic doublet results from the relaxation phenomenon due to the intrinsic finite-size effect [15]. 


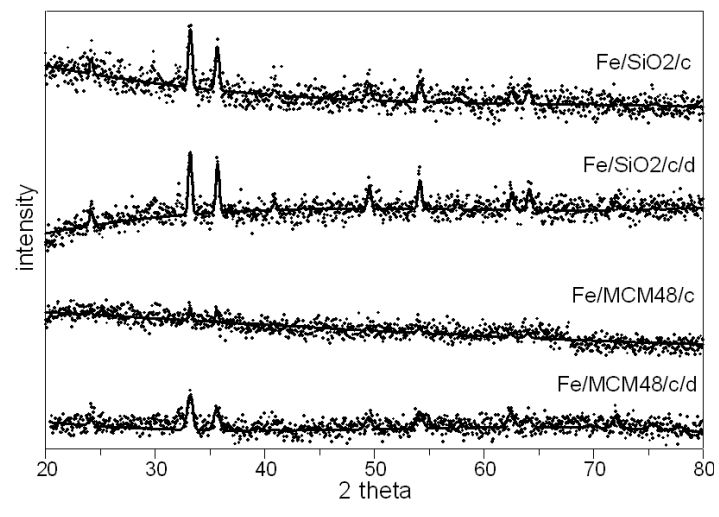

Fig. 4. The XRD diffraction patterns of $\mathrm{Fe} / \mathrm{SiO}_{2}$ and $\mathrm{Fe} / \mathrm{MCM} 48$ samples before and after silica removal.

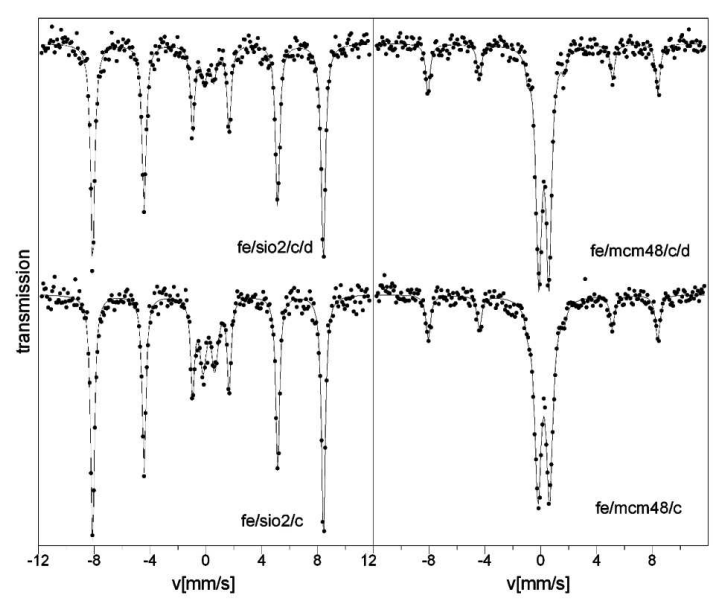

Fig. 5. ${ }^{57} \mathrm{Fe}$ Mössbauer spectra for $\mathrm{Fe}_{2} \mathrm{O}_{3}$ nanoparticles samples before and after template removal.

The relative contribution of superparamagnetic component is equal to $15(1) \%$ in the $\mathrm{Fe} / \mathrm{SiO} 2 / \mathrm{c}$ sample. After removal silica material the superparamagnetic component decreases to $8(2) \%$. It seems most likely that the smallest particles of hematite were removed together with the template. In the case of samples based on MCM48 matrix the relative contribution of superparamagnetic phase is dominating and achieves about $80 \%$. It results from significantly smaller pore sizes in MCM48 than those in $\mathrm{SiO}_{2}$ template. This contribution remains unchanged after MCM48 template removal. The presented experimental results indicate that the nanocrystalline form of iron oxide without MCM48 template is kept.

\section{Conclusions}

Iron oxides were prepared by the nanocasting technique. Two types of the silica materials were used.
Strongly dispersed $\mathrm{Fe}_{2} \mathrm{O}_{3}$ phases were formed in the pore systems of materials. The samples prepared by the application of the high-surface area matrices contained interconnected mesopores of the size around $3 \mathrm{~nm}$ showed larger surface area.

The TPR studies of the nature of the hematite evidenced differences in the redox properties of the species located on the different silica supports. Silica removal leads to the changes of iron-oxygen interactions. The MS studies confirm that iron oxide crystallites formed in MCM-48 template have mean sizes smaller than those obtained in $\mathrm{SiO}_{2}$ template. The matrix removal does not essentially influence on the hyperfine parameter values.

The nanocasting technique proved to be useful method to produce nanocrystalline powder without template.

\section{References}

[1] L. Weigang, Y. Dequan, S. Yan, G. Yun, X. Shuping, L. Hulin, Appl. Surf. Sci. 147, 39 (1999).

[2] V. Kesavan, D. Dhar, Y. Koltypin, N. Perkas, O. Palchik, A. Gedanken, S. Chandrasekaran, Pure Appl. Chem. 73, 85 (2001).

[3] T. Lindgren, H. Wang, N. Beermann, L. Vayssieres, E. Hagfeldt, S.E. Lindquist, Sol. Energy Mater. Sol. Cells 71, 231 (2002).

[4] Y.S. Hu, A. Kleiman-Shwarsctein, A.J. Forman, G.D. Stucky, E.W. McFarland, J. Phys. Chem. C 112, 15900 (2008).

[5] Y.S. Kang, S. Risbud, J.F. Rabolt, P. Stroeve, Chem. Mater. 8, 2209 (1996).

[6] R.F. Ziolo, E.P. Giannelis, B.A. Weinstein, M.P. O'Horo, B.N. Ganguly, V. Mehrotra, M.W. Russell, D.R. Huffman, Science 257, 219 (1992).

[7] F.M. Casula, P. Floris, C. Innocenti, A. Lascialfari, M. Marinone, M. Corti, R.A. Sperling, W.J. Parak, C. Sangregorio, Chem. Mater. 22, 1739 (2010).

[8] S.C. McBain, J. Dobson, Int. J. Nanomed. 3, 169 (2008).

[9] Y. Lu, Y. Yin, B.T. Mayers, Y. Xia, Nano Lett. 2, 183 (2002).

[10] V. Kesavan, D. Dhar, Y. Koltypin, N. Perkas, O. Palchik, A. Gedanken, S. Chandrasekaran, Pure Appl. Chem 73, 85 (2001).

[11] Q.F. Liu, C.X. Gao, J.J. Xiao, D.S. Xue, J. Magn. Magn. Mater. 260, 151 (2003).

[12] D.R. Cornejo, E. Padron-Hernandez, J. Magn. Magn. Mater. 316, e48 (2007).

[13] L. Wang, L. Wang, J. Zhang, J. Mater. Sci. 44, 6512 (2009).

[14] W. Gac, J. Goworek , G. Wójcik, L. Kępiński, Adsorption 14, 247 (2008).

[15] P.M. Déjardin, W.T. Coffey, Hyperfine Interact. 163, 73 (2005). 